\title{
TRIAGEM DE PROCESSAMENTO AUDITIVO CENTRAL EM CRIANÇAS DE 6 A 11 ANOS'
}

\author{
SCREENING CENTRAL AUDITORY PROCESSING IN \\ 6-11-YEAR-OLD CHILDREN
}

Fernando C. Capovilla ${ }^{1}$

CAPOVILLA, F. C. Triagem de processamento auditivo central em crianças de 6 a 11 anos. Rev. Bras. Cresc. Desenu Num., S. Paulo 12 (2), 2002.

Resumo: O artigo define processamento auditivo central e explica a sua importância para a aquisição de leitura e escrita. Explica, também, a natureza do distúrbio de processamento auditivo central e sua etiologia no histórico de otites repetidas durante o desenvolvimento da linguagem, bem como o seu envolvimento nos distúrbios processamento fonológico que subjazem aos problemas de linguagem oral e escrita, como ocorre na dislexia do desenvolvimento. Ressalta a necessidade de instrumentos para triagem de crianças com distúrbio de processamento auditivo central, com vistas a permitir experimentas dedicados ao teste de tratamentos para prevenção e remediação de distúrbios de linguagem oral e escrita. Examina a bateria de ZAIDAN (2001 ) para triagem de distúrbio de processamento auditivo central, desenvolvida no mestrado em Neurociências da USP. Trata-se de uma bateria de aplicação rápida em 15 minutos, e-composta de três testes de repetição de fala ouvida em condições de difícil audibilidade: fala distorcida com corte de frequências elevadas, fala contra ruído de fundo, e falas competitivas em escuta dicótica. Reanalisando os dados brutos daquela dissertação, este artigo sugere que a aparente inconclusividade dos achados sobre a sua validade deveu-se simplesmente à escolha de estatísticas inferenciais inadequadas ao delineamento. Reconduzindo as análises com estatísticas inferenciais apropriadas (i.e., Ancovas para controlar o efeito da ampla variação etária sobre os escores), o artigo demonstra que a bateria é, de fato, válida e sensível o suficiente para discriminar entre crianças de 6 a I I anos com desenvolvimento normal e aquelas com diagnóstico clínico de distúrbio de processamento auditivo central. Adicionalmente, descobriu que, além da bateria como um todo, cada um dos três testes que a compõem é capaz de, por si só, discriminar entre os grupos, identificando crianças com distúrbios de processamento auditivo central. Isto corrobora a adequação das listas de palavras e de sua gravação, revelando todo o esmero com que a bateria foi elaborada. Assim, o Brasil passa a dispor de um poderoso instrumento para a detecção de crianças com distúrbio de processamento auditivo central que estão em risco de fracassar na aquisição competente de leitura e escrita e que podem receber intervenção precoce.

Palavras-chave: processamento auditivo central; distúrbio de aprendizagem; dislexia.

Um dos fatores subjacentes às dificuldades de aquisição de leitura e escrita dos escolares vem sendo referido como distúrbio de processamento auditivo central (KATZ \& WILDE, 1989; KRAUS et al., 1996; MACFAR LAND \&
CACACE, 1995, 1998). De fato, num dos estudos de KATZ (1992), dentre 95 crianças com distúrbio de aprendizagem, 93 apresentaram distúrbio de processamento auditivo central. O processamento auditivo central diz respeito à sé-

Apoio: CNPq e FAPESP.

1 Psicólogo, Mestre em Psicologia pela Universidade de Brasília. Ph. D. em Psicologia Experimental pela Temple University of Philadelphia. Livre Docente em Neuropsicologia Clínica pela Universidade de São Paulo. Professor Associado, Instituto de Psicologia, Universidade de São Paulo. E-mail: capovilla@usp.br 
rie de processos de de codificação e transformação de informações das ondas sonoras, desde a orelha externa, passando pelas vias auditivas até o córtex. Ele envolve a detecção e interpretação de sons, a capacidade de identificar eventos sonoros quanto ao local, espectro, amplitude e tempo, além da realização de figura-fundo (e.g., identificação de sinal no ruído), e do reconhecimento, categorização, e atribuição de significado às informações acústicas (PHILLIPS, 1995).

Segundo a American Speech-Language and Hearing Association (1996), a habilidade de processamento auditivo central diz respeito às habilidades de localização e lateralização sonora, discriminação auditiva e reconhecimento de padrões acústicos, bem como de padrões auditivos temporais como resolução, mascaramento, integração e ordenação temporal, além do desempenho auditivo em presença de sinais acústicos degradados (i.e., distorcidos) ou competitivos. Como a fala consiste numa série de eventos acústicos codificados em termos de freqüência, intensidade e duração (DAVIS \& MCCROSKEY, 1980), a habilidade de resolução temporal é essencial à percepção da fala (KRAUS et al., 1995), e a de integração das pistas acústicas é vital à compreensão da fala (BALEN, 1997). De fato, CAPO-VILLA e CAPOVILLA (2001) descobriram que, na primeira série, crianças com habilidade de leitura silenciosa rebaixada (1 desvio-padrão abaixo da média) apresentam rebaixamentos significativos nas habilidades de discriminação fonêmica, memória de trabalho fonológica, e velocidade de processamento fonológico.

Segundo KATZ e WILDE (1989), as habilidades de processamento auditivo central desenvolvem-se até os 10 ou 12 anos de idade. Segundo ALVAREZ, CAETANO e NASTAS (1997), a etiologia dos distúrbios de processamento auditivo central inclui otites freqüentes na primeira infância, febres altas e contínuas, distúrbios específicos do desenvolvimento da função auditiva, pequenas lesões nas vias de condução, e privação sensorial durante a primeira infância. Uma história de otites médias também foi identificada em crianças portadoras desse distúrbio por CHER-MAK e MUSIEK (1997), que o relacionam a distúrbios de aprendizagem e do desenvolvimento da linguagem, e ao transtorno do déficit de atenção ou hiperatividade. De acordo com os pesquisadores, a privação auditiva intermitente decorrente de episódios repetidos de otite média antes dos dois anos de idade produz um efeito tampão de perda auditiva temporária no periodo crítico do desenvolvimento da linguagem (KATZ \& WILDE, 1989), o que predispõe a criança ao desenvolvimento de distúrbio de processamento auditivo central que pode persistir a vida toda, prejudicando as habilidades de compreender informações auditivas e o desempenho acadêmico (DOWNS, 1985). Tal privação parece produzir mudanças neuroanatomofisiológicas (BRANDES \& EHRINGER, 1981) que afetam a habilidade de perceber a fala em presença de ruído (PILLSBURY, GROSE \& HALL, 1991) e rebaixam o desempenho de reconhecimento de sentenças no ruído, a menos que a relação sinalruído seja muito elevada (GRAVEL \& WALLACE, 1992).

Segundo KEITH \& PENSAK (1991), o distúrbio de processamento auditivo central consiste numa inabilidade de atentar, discriminar, reconhecer, recordar ou compreender informações auditivas. Tal inabilidade é verificada apesar da ausência de comprometimento de inteligência geral e de audição periférica. Crianças com distúrbio de processamento auditivo central apresentam maior dificuldade em compreender a fala em presença de ruído de fundo, maior distratibilidade, atenção reduzida, dificuldade de comunicação, e baixo desempenho acadêmico, que é incompatível com seu nível de inteligência geral (CHER-MAK \& MUSIEK, 1992; GORDON \& WARD, 1995). Elas apresentam dificuldades de recepção da linguagem que se revelam numa menor habilidade de memória auditiva, de apreensão do sentido principal de enunciados, e de interpretação de palavras, frases, anedotas, metáforas, trocadilhos e de analogias ambíguas. Além disso, elas também apresentam dificuldades de produção de linguagem, que se revelam numa redução na taxa de emissão verbal e de recuperação lexical (BELLIS, 1996; CRUZ \& PEREIRA, 1996; FERRE, 1997).

De acordo com KEITH (1988), essas crianças são, em sua maioria, meninos, e seus limiares auditivos são normais, embora sua resposta a estímulos auditivos seja inconsistente. Elas apresentam alteração na atenção concentrada, fatigam-se facilmente frente à tarefas complexas ou prolongadas, distraem-se com facilidade, mostram sensibilidade exacerbada a sons intensos, têm dificuldade em seguir instruções verbais, solicitam freqüentes repetições da informação auditiva, têm dificuldade de memorização, de recitação da tabuada e do alfabeto, demoram a responder, têm dificuldade em aprender as relações grafofonêmicas, e em compreender piadas e linguagem figurada. A dificuldade de compreensão da fala é maior em presença de ruído de fundo, de fala simultânea, ou no acompanhamento da fala de mais de dois interlocutores. Tais pré-escolares tendem a preferir um estilo de aprendizagem cinestésico, 
enquanto que os pré-escolares sem distúrbio preferem um estilo mais auditivo e verbal.

A habilidade de processamento auditivo central pode ser avaliada tanto por medidas eletrofisiológicas quanto por testes comportamentais (MUSIEK \& BARAN, 1987). Os testes comportamentais sempre usam como estratégia a redução da redundância do material apresentado à audição da criança, em termos das pistas acústicas, sintáticas, semânticas e linguísticas no sinal ou mensagem (BELLIS, 1996; FERRE, 1997; SCHOCHAT, 1998). Eles permitem diagnóstico diferencial das habilidades auditivas específicas que se encontram comprometidas (CARVALHO, 1997), mas sua validade e confiabilidade requer que o avaliando tenha suficiente audição periférica, nivel cognitivo, e linguagem receptiva e expressiva (BELLIS, 1996; CHERMAK \& MUSIEK, 1997; FERRE, 1997). Tais testes compõem baterias, sendo que cada um dos testes avalia pelo menos duas das habilidades de atenção auditiva, discriminação e fechamento auditivo, interação binaural, integração e associação de sinais, e processamento temporal (FERRE, 1997). Como o comprometimento pode ser específico e mostrarse apenas em um teste e não nos outros (BARAN \& MUSIEK, 1999), é essencial usar baterias de testes e não apenas um ou outro teste isolado (HOOD \& BERLIN, 1996). Além disso, como lembra KEITH (1986), é preciso demonstrar que a bateria é válida (i.e., que ela mede aquilo que objetiva medir, ou seja, que seus resultados correspondem aos de outros instrumentos e da observação clínica) e que ela é também confiável (i.e., que seus resultados inostrainse estáveis em avaliações repetidas). Finalmente, feito isto, é preciso obter tabelas de dados normativos do desempenho nos subtestes das baterias para cada faixa etária, já que em escolares o desempenho auditivo nesses testes melhora com a idade (MUSIEK \& LAMB, 1999).

Embora ainda não haja dados epidemiológicos sobre a incidência de distúrbios de processamento auditivo central, de acordo com pesquisadores (BELLIS, 1996; CHERMAK \& MUSIEK, 1997; LEWIS, 1986), 70\% das crianças até 3 anos de idade já tiveram um ou mais episódios de otite média, sendo que mais de $30 \%$ tiveram três ou mais. Além disso, 10\% dos escolares apresentam distúrbios de aprendizagem; 10-20\% apresentam transtorno do déficit de atenção, sendo que 25-50\% deles apresentam distúrbios de aprendizagem; e 70-80\% das crianças que apresentam distúrbio de aprendizagem e de processamento auditivo central também apresentam distúrbio do desenvolvimento da linguagem.
A ausência de levantamentos epidemiológicos da incidência de distúrbios de processamento auditivo central se deve à falta de instrumentos de triagem das habilidades de processamento auditivo central. Por isso, com o objetivo de fòmecer um instrumento de triagem capaz de identificar rápida e facilmente crianças com transtornos de processamento auditivo central, e inspirada na bateria SCAN (Screening Test for Auditory Processing Disorders) de KEITH (1986), ZAIDAN (2001) dedicou-se à elaboração de uma bateria de testes de triagem do processamento auditivo central em sua dissertação de mestrado em Neurociências e Comportamento na Universidade de São Paulo. A triagem objetiva apenas identificar crianças que podem apresentar alteração no processamento auditivo central, ou seja, detectar as crianças que necessitam de avaliação para fins diagnósticos, mas não fornecer o diagnóstico propriamente dito. A triagem contribui para diminuir avaliações desnecessárias, reduzindo desperdícios de recursos materiais e humanos, e auxiliando a adequar os delineamentos educacionais às características da populaçào escolar.

Além de válida e fidedígna, a triagem deve ser um procedimento uniforme e padronizado, bem aceito pelos avaliandos , e de aplicação e interpretação simples, fácil e rápida em ambiente natural (BLSS \& HULMES, 1998; CHERRY, 1992). Coerente com tais objetivos, a bateria de triagem de ZAIDAN (2001) é composta por apenas três testes, contrastando com baterias de avaliação para um exame mais detido e extenso, como a de PEREIRA e SCHOCllAT (1997b) que tem 12 testes: 1) Fala no ruído (SCHOCHAT \& PEREIRA, 1997), 2) Baixa redundância: Fala filtrada e fusão biaural (PEREIRA \& SCHOCHAT, 1997a), 3) PSI em português (ZILIOTTO, KALIL \& ALMEIDA, 1997), 4) SSI em português (KAI,IL, ZlLIOTTO, \& ALMEIDA,1997), 5) Consoante-vogal de escuta direcionada (TEDES-CO), 1997), 6) Escuta com dígitos (SANTOS, M. F., \& PEREIRA, 1997), 7) Nào-verbal de escuta direcionada (ORTIZ \& PEREIRA, 1997), 8) Sons ambientais competitivos CES (SCHOCHAT, 1997), 9) Dissílabos alternados SSW (BORGES, 1997a), 10) Sentenças para avaliar reconhecimento da fala (COSTA MORIO, \& MANGABEIRA ALBERNAZ, 199-b), 11) Consciênca fonológica (SANTOS, M. T., \& PEREIRA, 1997), 12) Percepção auditiva para deficientes auditivos com palavras MTS (BORGES, 1997b). 


\section{A BATERIA SCAN (SCREENING TEST FOR AUDITORY PROCESSINC DISORDERS) DE KEITH (1986)}

A bateria de Zaidan (2001) é inspirada na bateria SCAN (Screening Test fòrAudito^/y ProcessingDisorders) de Keith (1986), uma das baterias comportamentais mais emprcgadas por audiologistas nos Estados Unidos, embora seja criticada por carecer de teste de processamento temporal e de validação com cérebro-lesados (Chermak et al., 1998) e por produzir efeito de carreamento (Amos \& Humes, 1998). A bateria SCAN requer apenas um aparelho de som estereofónico portátil e dois conjuntos de forte de ouvido, e pode ser aplicada numa sala silenciosa em apenas 20 minutos. Foi normalizada na t‘aixa etária de 3 a 11 anos de idade. Apresenta listas de palavras monossílabas familiares e de fácil reconhecimento a crianças de primeira séne, incluindo substantivos, adjetivos, verbs e pronomes. SCAN compõe-se de três testes:

1) Escuta dicótica de palavras competitivas: Repetição de 108 palavras alvo monossilabas (i. c., 2 listas de 25 pares de palavras cada uma, mais 8 itens de prática) apresentadas dicoticamente (i.e., em cada par de palavras, uma é apresentada à orelha esquerda e a outra à direita, ambas simultaneamente ou com diferença de no máximo 5 milésimos de segundo). Nesse teste, a criança é solicitada a ouvir duas palavras simultaneamente e a repetir ambas. Segundo BELLIS (1996) e FERRE (1997), este teste avalia as habilidades de atenção dividida, integração binaural, discriminação, associação, recuperação e organização. Diferentes testes variam o material apresentado, como dígitos, rimas (MUSIEK et al., 1989), e consoante-vogal (BERLIM et al., 1972).

2) Escuta de fala filtrada passa-baixo: Repetição de 44 palavras monossílabas ouvidas em apresentação monaural com atenuação de freqüências elevadas (i.e., cortes a partir de $1.000 \mathrm{~Hz}$ ). Segundo MACHADO (1996), tal corte dificulta a identificação dos fonemas consonantais, especialmente os fricativos que têm maior concentração de componentes de alta freqüência. Segundo KEITH (1986), este teste avalia a habilidade de decodificar a fala em situação de baixa redundância e de realizar fechamento auditivo do sinal. Diferentes testes variam a freqüência de corte em vários níveis como $400 \mathrm{~Hz}, 500 \mathrm{~Hz}$, ou outra.

3) Escuta de fala no ruído: Repetição de 44 palavras-alvo monossílabas (i.e., 2 listas de 22 palavras cada uma) apresentadas monoauralmente contra rumor de conversa de fundo, sendo a relação sinal ruído de $+8 \mathrm{~dB}$. Sinal e ruído podem ser apresentados ambos na mesma orelha (ipsilateralmente), ou cada qual numa orelha diferente (contralateralmente). Esse teste avalia a dificuldade em compreender fala contra ruídos de fundo, que é uma das queixas mais freqüentes de crianças com problemas de aprendizagem. Ele avalia as habilidades de atenção seletiva e sustentada, fechamento auditivo e decodificação de fala de baixa redundância. Diferentes testes variam a relação sinal ruído, sendo que a inteligibilidade é tão maior quanto maior for a diferença do sinal para o ruído em dB (PEREIRA, 1993), embora tal diferença normalmente fique entre 0 e $10 \mathrm{~dB}$. Diferentes testes também variam no tipo de ruído de fundo (e.g., ruído branco, burburinho, cafeteria), sendo que quanto maior for a semelhança entre sinal e ruído, tanto mais difícil será o teste.

\section{A BATERIA DE ZAIDAN (2001)}

A bateria de ZAIDAN (2001) tem apenas três testes, todos envolvendo repetição de palavras sob escuta difícil, com um tempo total de aplicação de 15 minutos:

1) Teste de escuta de fala $f$ Itrada passa-baixo que dura 4 minutos e 16 segundos e apresenta 44 palavras (sendo 4 de treino e 40 de teste) separadamente, 20 a cada orelha, e filtradas com corte de frequências acima de $1.000 \mathrm{~Hz}$;

2) Teste monótico de escuta de fala no ruido que dura 4 minutos e 7 segundos e apresenta 44 palavras (sendo 4 de treino e 40 de teste, 20 apresentadas à orelha direita e 20 à esquerda), com ruído de conversação filtrado eletronicamente (i.e., sem nenhuma palavra identificável) e relação sinal-ruido de $+5 \mathrm{~d} \wedge \mathrm{B}$ (i.e., o sinal tem intensidade $5 \mathrm{~dB}$ superior à do ruído);

3) Teste de escuta dicótica de palavras competitivas que dura 6 minutos e 37 segundos, e apresenta 108 palavras (i.e.,4 pares de treino e 50 pares de teste), sendo as palavras do par apresentadas simultaneamente, cada palavra numa orelha (e sendo que as palavras têm a mesma acentuação tônica e duração, com coincidência nas concentrações de energia dos espectros).

Em cada teste há três listas de palavras dissílabas paroxítonas que a criança deve repetir sob situação de escuta difícil. Respeitando critérios de elaboração de materiais de avaliação de linguagem, foram escolhidas palavras familiares à idade dos avaliandos (para evitar efeito de vocabulário), e com extensão controlada e distribui- 
ção fonética representativa da língua portuguesa. Além disso, foi feito um certo esforço para levar em consideração a distribuição de freqüência de ocorrência das palavras do Minidicionário Aurélio como função da classe gramatical (58\% são substantivos, 23\% são adjetivos, 17\% são verbos, 1,5\% são advérbios, adjetivos e pronomes), número de sílabas (34,5\% são trissílabos, 30\% são tetrassílabos, $15 \%$ são bissílabos, $15 \%$ são pentassílabos, e $0,6 \%$ são inonossílabos), e tonicidade (54\% são paroxítonos, 35\% são oxítinos, e 11\% são proparoxítonos).

A partir do material de leitura das crianças de primeira série, foram escolhidas palavras bissílabas paroxítonas, a maioria correpondendo a substantivos, seguidas de adjetivos, verbos e pronomes. Para balanceamento fonético, foi empregado como critério a distribuição de freqüência de ocorrência dos segmentos fônicos do léxico do Minidicionário Aurélio. Em cada uma das listas, o índice de correlação entre a distribuição de frequência de ocorrência dos segmentos fônicos da lista e os do dicionário sempre foi maior que $r=0,95$. Após o balanceainento, as listas tòram submetidas a juizes, e tòram eliminadas as palavras com alta freqüência de acerto, erro ou substituições. Depois disso, após novo balanceamento, as listas foram gravadas em estúdio profissional em formato digital e, por meio do software Wave, foram removidos os picos de amplitude de modo a estreitar e uniformizar a faixa dinâmica, controlando artefatos audíveis. As três listas empregadas encontram-se no Quadro 1.

Quadro 1: Listas de palavras empregadas nos testes da bateria de triagem

\begin{tabular}{|c|c|c|c|c|c|c|}
\hline \multicolumn{2}{|c|}{ fala filtrada } & \multicolumn{2}{c|}{ fala no ruído } & \multicolumn{2}{c|}{ palavras competitivas } \\
\hline OD & OE & OD & OE & ordem & OD & OF \\
\hline Bode & suco & disse & Trave & 1 & bola & time \\
\hline Milho & dente & pano & Dança & 2 & zero & milho \\
\hline nave & pura & noite & Missa & 3 & ninho & fundo \\
\hline fundo & cesta & gorda & Roça & 4 & lente & nunca \\
\hline lado & grade & risco & Fibra & 5 & quente & pulga \\
\hline louco & manga & corpo & Gente & 6 & gripe & pista \\
\hline minha & riso & nunca & Lixo & 7 & susto & mundo \\
\hline uva & rua & zebra & Soro & 8 & manda & cinto \\
\hline pires & tira & circo & Manda & 9 & sete & gorro \\
\hline sino & cinto & verde & Lente & 10 & dente & verde \\
\hline
\end{tabular}

\section{OS ESTUDOS DE ZAIDAN}

ZAIDAN (2001) conduziu dois estudos, o primeiro para testar a adequação das três listas de palavras que compõem a bateria e o segundo para testar a validade da bateria. $\mathrm{O}$ primeiro estudo envolveu 90 crianças de 6 a 11 anos de idade e teve duas etapas, a primeira com 30 crianças para eliminar palavras inadequadas das listas, e a segunda com 60 crianças para checar se as novas listas estavam aceitáveis. Como esta segunda etapa produziu resultados aceitáveis, as 60 crianças foram aproveitadas como grupo controle e seus dados foram usados para o segundo estudo. Todas as 90 crianças avaliadas no primeiro estudo eram destras, estudavam em escola particular e apresentavam níveis de audição normais e simétricos nas duas Grelhas (i.e., limiares auditivos até IS $\mathrm{dBN} \wedge \mathrm{A}$ ), além de ausência de alterações de linguagem, fala, aprendizagem e processamento auditivo central, conforme dados de anamnese e escores no Perfil de Habilidades Fonológicas (CARVALIIO, ALVAREZ, \& CAETANO, 1998).

O segundo estudo usou os resultados dessas 60 crianças controle (10 crianças de cada faixa etária, dos 6 aos 11 anos) como critério contra o qual comparar os resultados de 11 outras crianças com diagnóstico clínico de distúrbio de processamento auditivo central e idades variando de 6 anos e 4 meses a 11 anos e 11 meses. O objetivo do estudo era testar a validade da bateria por meio da comparação dos escores entre os dois grupos (i.s., o grupo experimental de 11 crianças previamente diagnosticadas como tendo distúrbio de processamento auditivo central e o grupo controle de 60 crianças sem esse diagnóstico) com vistas a verificar se a bateria toda, ou pelo menos um ou dois de seus três testes, seria capaz de discriminar entre esses dois grupos.

\section{RESULTADOS ORIGINAIS RELATADOS}

Quanto à qualidade do material, foi observado que as três listas de palavras mostraramse foneticamente representativas do português, uma vez que o coeficiente de correlação não paramé-trico de Spearman entre as freqüências de distribuição dos fonemas nelas e no corpus de palavras do Minidicionário Aurélio variou de 0,979 a 0,997 .

Quanto à distribuição de erros como função da faixa etária, não houve análise de dados. Apesar do claro declínio no número médio de erros com a idade que aparece nas Tabelas 4 a 8 da dissertação, nenhuma análise foi feita para avaliar se a bateria (ou qualquer um de seus testes) 
efetivamente discriminaria entre séries sucessivas. A resposta a esta pergunta requereria análise de variância (Anova) seguida de análises de comparação entre pares. Contudo, em vez disso, foi apenas aplicado o teste Runs para avaliar se houve tendência de declínio (e. g., por fadiga) ou aumento (e.g., por aprendizagem) no acerto ao longo das três listas, sendo os resultados negativos.

Quanto à capacidade dos testes de discriminar entre os grupos experimental e controle, curiosamente, nenhum teste estatístico foi conduzido. Aparentemente, a variação dos escores dentro dos grupos (que, conforme esperado pela ampla variação etária de 6 a 11 anos, foi muito grande) acabou chamando mais atenção do que a variação entre os grupos (i.e., a variação intragrupo mostrou-se maior do que a entre-grupos, como seria esperado, dada a ampla variação etária). Assim, em vez de comparar os escores entre os grupos, as análises limitaram-se a comparar os escores dentro de cada grupo. Ou seja, dada a forte variação de escores intra-grupo devido à ampla variação etária (6 a 11 anos), as análises limitaram-se a regressões lineares elementares do efeito geral da idade sobre o desempenho em cada grupo e em cada teste, perdendo-se por completo o objetivo central do estudo, que foi o de verificar se a bateria (ou qualquer um de seus testes) seria ou não capaz de discriminar entre os grupos experimental e controle.

Para obter a resposta a esta questão central que motivou o estudo (i.e., a questão de se há ou não diferença discriminável entre os grupos) é necessário comparar os escores entre os grupos. Para fazê-lo, contudo, dada a grande variação nos escores dentro de cada um dos grupos, esperada devido à ampla variação etária, seria necessário controlar o efeito da idade sobre a variação dos escores dentro de cada grupo. Isto poderia ter sido feito simplesmente por meio de análise de covariância (Ancova), em que o efeito do tipo de grupo sobre o escore nos testes seria avaliado a partir do controle, como covariante, do efeito da idade da criança.

Quanto à confiaóilidade do teste, em vez de ter sido calculada a correlação entre os escores de teste e reteste, foi calculado se haveria diferença significativa entre os escores da primeira para a segunda aplicação a 30 crianças do grupo controle (i.e., sem distúrbios diagnosticados). Os resultados mostraram-se significativos em todos os três testes, ou seja, os desempenhos na segunda aplicação foram significativamente diferentes daqueles da primeira aplicação, o que depõe contra a confiabilidade ou fidedignidade da bateria. Se há diferença nos escores entre a primeira e a segunda aplicações, isto significa que os resulta- dos não são confiáveis. Contudo, comparando as médias da primeira à segunda aplicações, como não há qualquer ganho ou perda aparente, isto sugere que os escores variaram de maneira espúria entre as aplicações. Ou seja, enquanto alguns aumentaram da primeira à segunda, outros diminuíram, sendo que os grupos como um todo não apresentaram qualquer tendência, mas apenas variações espurias intra-grupo.

Assim, infelizmente, devido ao relativamente baixo número de crianças do grupo experimental $(\mathrm{N}=11)$ e à grande variação da faixa etária (entre 6 e 11 anos), mas principalmente ao emprego de estatísticas descritivas e inferenciais não-paramétricas inadequadas à questão levantada no estudo e à natureza do delineamento experimental empregado, os resultados daquele estudo não foram conclusivos.

\section{RESULTADOS REANALISADOS}

\section{EFEITO DA FAIXA ETÁRIA. REANÁLISES ESTATÍSTICAS LEVANDO EM CONSIDERAÇÃO AS 60 CRIANÇAS DO GRUPO CONTROLE}

A Figura 1 representa os escores médios no teste de repetição de fala filtrada como função da faixa etária. Conforme a figura, houve uma tendência geral de alta na frequência de acertos como função da idade.

Anova do escore de repetição de fala filtrada como função de faixa etária revelou efeito significativo, F $(5,54)=8,77$, p < 0,000. Análises de comparação de pares de Bonferroni revelaram que crianças de 6 anos tiveram desempenho abaixo de todas as demais, que crianças de 5 anos tiveram desempenho inferior ao das de 10 anos. Ou seja, o teste de fala filtrada foi capaz de discriminar entre crianças de 6 anos e as demais. Análises de comparação de pares de Fisher LSD revelaram que crianças de 6 anos tiveram desempenho abaixo de todas as demais crianças; que crianças de 7 anos tiveram desempenhos abaixo do das crianças de 10 anos; e que crianças de 9 anos tiveram desempenho abaixo do das de 10 anos; e que as crianças de 10 anos tiveram desempenho abaixo do das de 11 anos. Ancova do escore de repetição de fala filtrada como função do sexo das crianças, tendo como covariante a idade falhou em revelar efeito significativo do sexo das crianças, mas revelou efeito do covariante idade das crianças, F ( I, 57) = 15 .60, p < 0,000. Ou seja, os desempenhos de meninos e meninas no teste de fala filtrada podem ser considerados equivalentes entre si. 
Figura 1: Escores médios no teste de repetição de fala filtrada como função da faixa etária da amostra de 60 crianças do grupo controle (i.e., sem diagnóstico de distúrbio de processamento auditivo central).

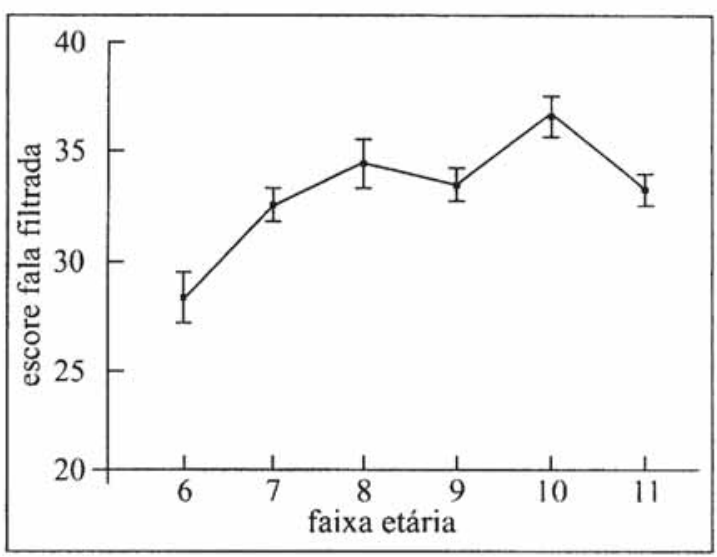

A Figura 2 representa os escores médios no teste de repetição de fala no ruido como função da faixa etária. Conforme a figura, houve uma tendência geral de alta na frequêência de acertos como função da idade.

Anova do escore de repetição da fala no ruído como função de faixa etária revelou efeito significativo, F $(5,54)=5,36, p<0,000$. Análises de comparação de pares de Bonferroni revelaram que crianças de 6 anos tiveram desempenho abaixo dos das crianças de 10 e 11 anos. Análises de comparação de pares de Fisher LSD revelaram que crianças de 6 anos tiveram desempenho abaixo de todas as demais crianças; que

Figura 2: Escores médios no teste de repetição de fala no ruído como função da faixa etária da amostra de 60 crianças do grupo controle (i.e., sem diagnóstico de distúrbio de processamento auditivo central)

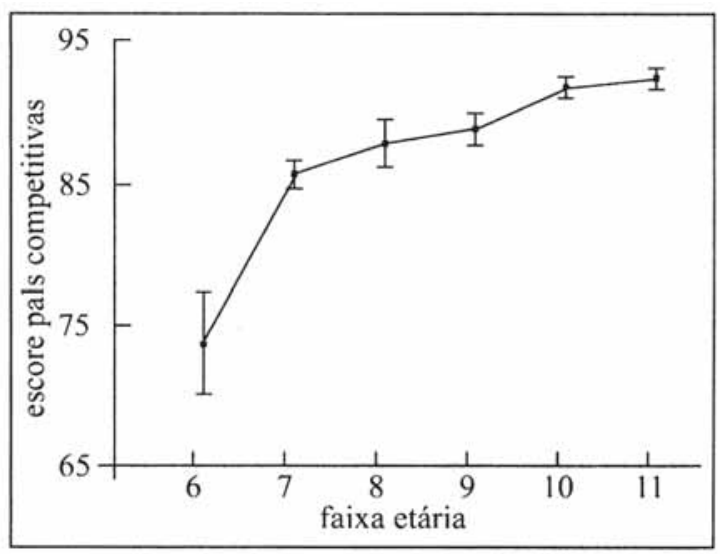

crianças de 7 anos tiveram desempenhos abaixo dos das crianças de 10 e 11 anos; e que crianças de 8 e de 9 anos tiveram desempenho abaixo das de 11 anos. Ancova do escore de repetição da fala filtrada como função do sexo das crianças, tendo como covariante a idade falhou em revelar efeito significativo do sexo das crianças, mas revelou efeito do covariante idade das crianças, $F(1,57)=21,05, p<0,000$. Ou seja, os desempenhos de meninos e meninas no teste de fala no ruído também podem ser considerados equivalentes entre si.

A Figura 3 representa os escores médios no teste de repetição de palavras competitivas como função da faixa etária. Conforme a figura, houve uma tendência geral de alta na frequêencia de acertos como função da idade.

Figura 3: Escores médios no teste de repetição de palavras competitivas como função da faixa etária da amostra de 60 crianças do grupo controle (i.e., sem diagnóstico de distúrbio de processamento auditivo central)

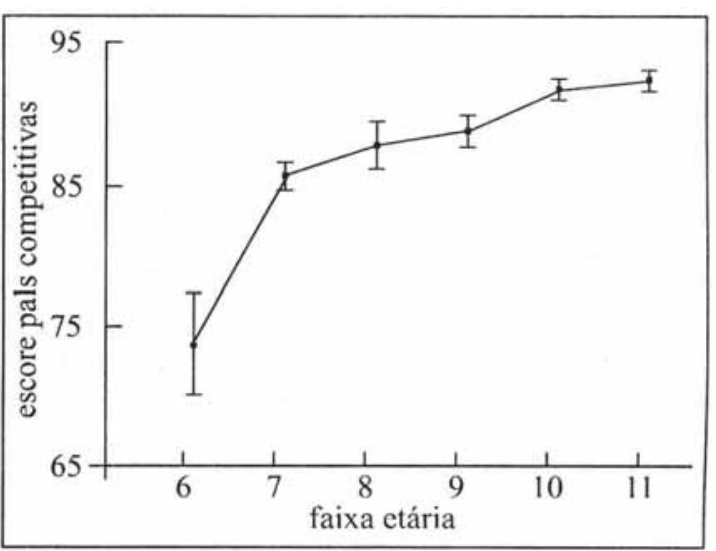

Anova de escore de repetição de palavras competitivas como função de faixa etária revelou efeito significativo, $\mathrm{F}(5,54)=14,41, \mathrm{p}<0,000$. Análises de comparação de pares de Bonferroni revelaram que crianças de 6 anos tiveram desempenho abaixo de todas as demais crianças. Análises de comparação de pares de Fisher LSD revelaram que crianças de 6 anos tiveram desempenho abaixo de todas as demais crianças; que crianças de 7 anos tiveram desempenhos abaixo dos das crianças de 10 e 11 anos. Ancova do escore de repetição da fala filtrada como função do sexo das crianças, tendo como covariante a idade falhou em revelar efeito significativo do sexo das crianças, mas revelou efeito do covariante idade 
das crianças, $F(1,57)=45,00$,p $<0,000$. Ou seja, os desempenhos de meninos e meninas no teste de palavras competitivas também podem ser considerados equivalentes entre si.

A Figura 4 representa os escores médios na bateria de processamento auditivo central como função da faixa etária. Conforme a figura, houve uma tendência geral de alta na freqüência de acertos como função da idade.

Anova do escore de repetição de palavras competitivas como função de faixa etária revelou efeito significativo, $\mathrm{F}(5,54)=20,55$, p $<0,000$. Análises de comparação de pares de Bonferroni revelaram que crianças de 6 anos tiveram desempenho abaixo de todas as demais crianças; e que crianças de 7 anos tiveram desempenho abaixo do das crianças de 10 e 11 anos de idade. Análi-

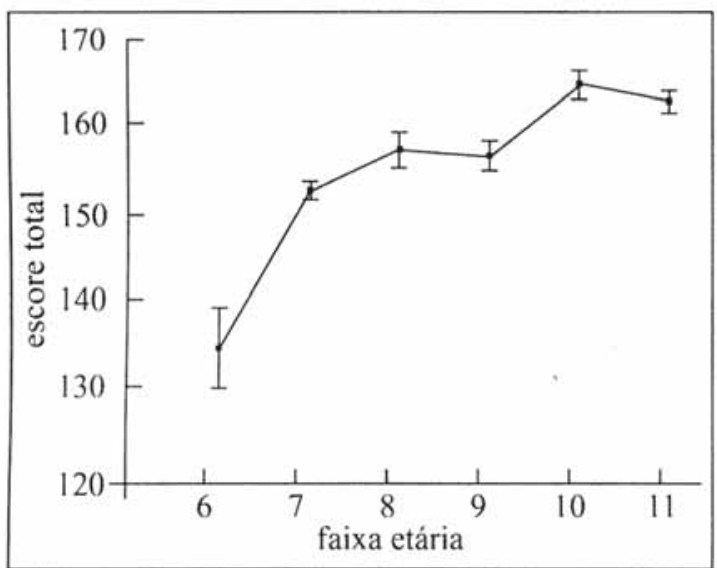

Figura 4: Escores médios na bateria de triagem de distúrbio de processamento auditivo central como função da faixa etária da amostra de 60 crianças do grupo controle (i.e., sem diagnóstico de distúrbio de processamento auditivo central)

ses de comparação de pares de Fisher LSD revelaram que crianças de 6 anos tiveram desempenho abaixo de todas as demais crianças; que crianças de 7 anos tiveram desempenhos abaixo dos das crianças de 10 e 11 anos; e que crianças de 9 anos tiveram desempenho abaixo do das crianças de 10 anos. Ancova do escore total como função do sexo das crianças, tendo como covariante a idade falhou em revelar efeito significativo do sexo das crianças, mas revelou efeito do covariante idade das crianças, $\mathrm{F}(\mathrm{I}, 57)=55,75, \mathrm{p}<0,000$. Ou seja, os desempenhos de meninos e meninas na bateria de triagem de distúrbios de processamento auditivo central, como um todo, também podem ser considerados equivalentes entre si.
Efeito do status "normal” versus "paciente”: Reanálises estatísticas considerando as 60 crianças do grupo controle e as 11 do grupo experimental.

A Figura 5 representa os escores médios no teste de fala filtrada como função do status das crianças (N: normal, P: paciente). Encontramse representadas as médias em fala filtrada das duas amostras, as 60 crianças normais (N) e as 11 crianças com diagnóstico de distúrbio de processamento auditivo central (P).

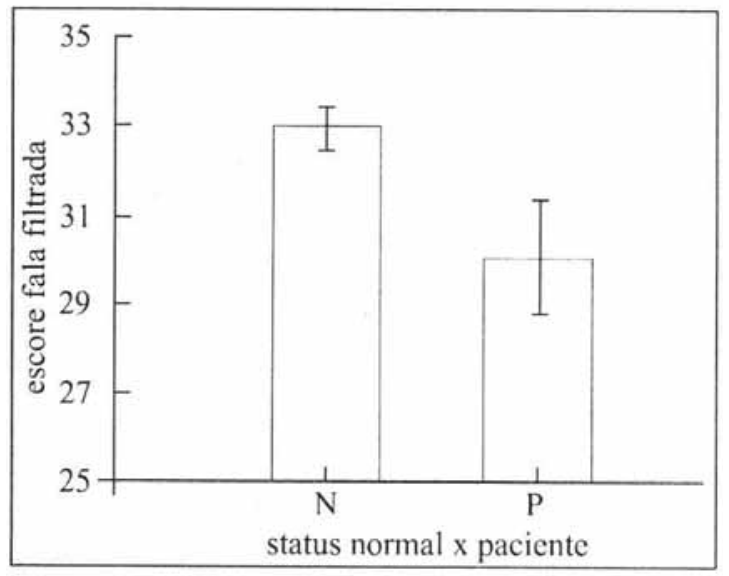

Figura 5: Escores médios no teste de fala filtrada como função do status das crianças (N: normal, $\mathrm{P}$ : paciente)

- Ancova do escore de fala filtrada como função do status ("normal” versas "paciente”) das 71 crianças, tendo como covariante a idade das crianças, revelou efeito significativo do status das crianças, F ( I, 68) = 10,29,p < 0,000, bem como do covariante idade das crianças, $F(1,68)=$ $16,51, \mathrm{p}<0,000$. Ou seja, uma vez controlada a variação produzida pela idade das crianças, notou-se que as crianças com diagnóstico de distúrbio de processamento auditivo central apresentaram, no teste de fala filtrada, escore significativamente inferior ao escore das crianças normais (i.e., sem esse diagnóstico). Assim, podese dizer que o teste de fala filtrada da presente bateria foi eficaz em identificar crianças com distúrbio de processamento auditivo central, discriminando-as das nonnais. As médias, ajustadas pela análise (diferenternente das médias não ajustadas representadas na figura), com respectivos errospadrão foram as seg uintes: Para as 60 crianças normais: 33,09 (e.p. $=0,45$ ), e para as 11 crianças com distúrbio de processamento auditivo central: 29,35 (e.p. = 1,07). 
A Figura 6 representa os escores médios no teste de fala no ruído como função do status das crianças (N: normal, P: paciente). Encontramse representadas as médias em fala no ruido das duas amostras, as 60 crianças normais $(\mathrm{N})$ e as 11 crianças com diagnóstico de distúrbio de processamento auditivo central (P).

Ancova do escore de fala no ruído como função do status ("normal” versus "paciente”) das 71 crianças, tendo como covariante a idade das crianças revelou efeito significativo do status das crianças, $\mathrm{F}(1,68)=61,68, \mathrm{p}<0,000$, bem como do covariante idade das crianças, $\mathrm{F}(\mathrm{I}, 68)=23,23$, $\mathrm{p}<0,000$. Ou seja, uma vez controlada a variação produzida pela idade das crianças, notou-se que as crianças com diagnóstico de distúrbio de processamento auditivo central apresentaram, no teste de fala no ruído, escore significativamente inferior ao escore das crianças normais (i.e., sem esse diagnóstico). Assim, pode-se dizer que o teste de fala no ruido da bateria de Zaidan foi eficaz em identificar crianças com distúrbio de processamento auditivo central, discriminando-as das normais. As médias, ajustadas pela análise (diferentemente das médias não ajustadas representadas pela figura), com respectivos erros-padrão foram as seguintes: Para as 60 crianças normais (do grupo controle): 35,06 (e.p. $=0,31$ ), e para as 11 crianças com distúrbio de processamento auditivo central (do grupo experimental): 28,76 (e.p. $=0,74)$.

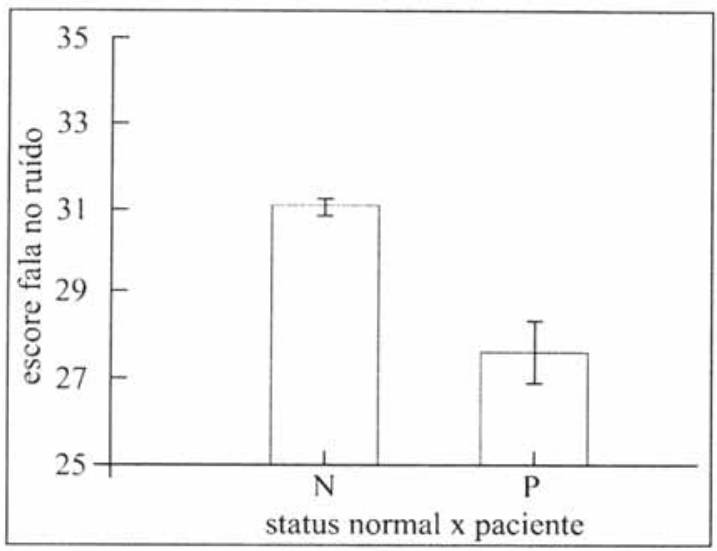

Figura 6: Escores médios no teste de fala no ruido como função do status das crianças ( $\mathrm{N}$ : normal, P: paciente).

A Figura 7 representa os escores médios no teste de palavras competitivas como função do status das crianças (N: normal, P: paciente). Encontram-se representadas as médias em palavras competitivas das duas amostras, as 60 crian- ças normais $(\mathrm{N})$ e as 11 crianças com diagnóstico de distúrbio de processamento auditivo central (P). Ancova do escore de palavras coinpetitivas como função do status ("normal” versus "paciente") das 71 crianças, tendo como covariante a idade das crianças revelou efeito significativo do status das crianças, $F(I, 68)=62,16, p<0,000$, bem como do covariante idade das crianças, $\mathrm{F}(1,68)=40,13, \mathrm{p}<0,000$. Ou seja, uma vez controlada a variação produzida pela idade das crianças, notou-se que as crianças com diagnóstico de distúrbio de processamento auditivo central apresentaram, no teste de palavras competitivas, escore significativamente inferior ao escore das crianças normais (i.e., sem esse diagnóstico). Assim, pode-se dizer que o teste de palavras competitivas da presente bateria foi eficaz em identificar crianças com distúrbio de processamento auditivo central, discriminando-as das normais.

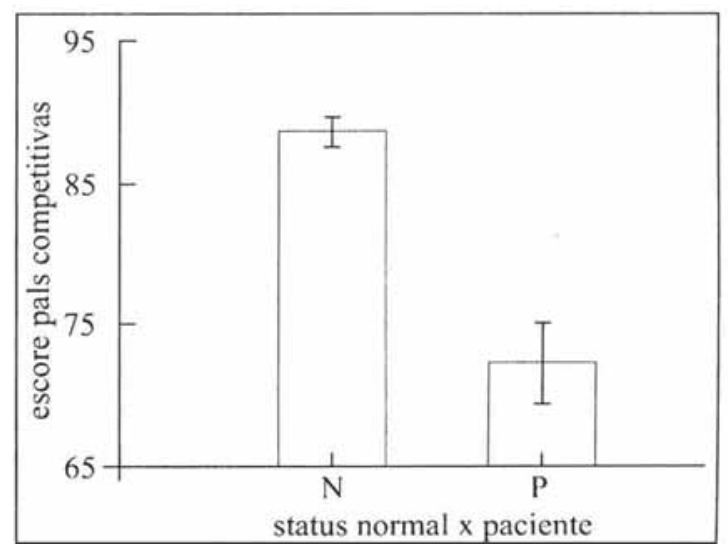

Figura 7: Escores médios no teste de palavras competitivas como função do status das crianças (N: normal, P: paciente).

As médias, ajustadas pela análise (diferentemente das médias não ajustadas representadas pela figura), com respectivos erros-padrão foram as seguintes: Para as 60 crianças normais: 87,10 (e.p. $=0,87$ ), e para as $1 \mathrm{l}$ crianças com distúrbio de processamento auditivo central: 69,52 (e.p. $=2,04)$.

A Figura 8 representa os escores totais na bateria de triagem de processamento auditivo central como função do status das crianças (N: normal, P: paciente). Encontram-se representados os escores totais das duas amostras, as 60 crianças normais $(\mathrm{N})$ e as 11 crianças com diagnóstico de distúrbio de processamento auditivo central (P).

Ancova do escore total como função do status ("normal” versus "paciente") das 71 crianças, tendo como covariante a idade das crianças 
revelou efeito significativo do status das crianças, $\mathrm{F}(1,68)=73,64, \mathrm{p}<0,000$, bem como do covariante idade das crianças, $F(1,68)=49,78$, $\mathrm{p}<0,000$. Ou seja, uma vez controlada a variação produzida pela idade das crianças, notou-se que as crianças com diagnóstico de distúrbio de processamento auditivo central apresentaram, na bateria como un todo, escore significativamente inferior ao score das crianças normais (i.e., sem esse diag nóstico). Assim, pode-se dizer que a bateria de triagem de distúrbio de processamento auditivo central de Zaidan foi eficaz em identifi car crianças com distúrbio de processamento au-

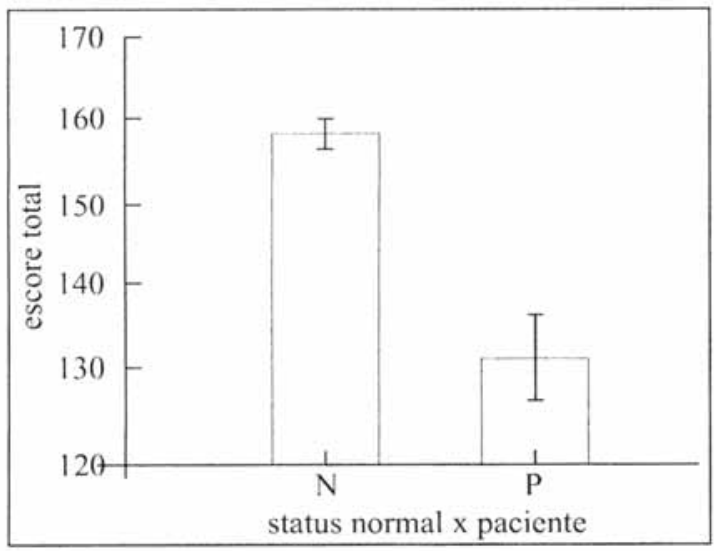

Figura 8: Escores médios na bateria de triagem de distúrbio de processamento auditivo central como função do status das crianças (N: normal, $\mathrm{P}$ : paciente)

ditivo central, discriminando-as das normais As médias, ajustadas pela análise (diferentemente das médias não ajustadas representadas pela figura), com respectivos erros-padrãc foram as seguintes: Para as 60 crianças nor mais: 155,23 (e.p. = 1,25) e para as $1 \mathrm{l}$ crianças com distúrbio de processamento auditivo central: 127,63 (e.p. = 2,95).

\section{CONCLUSÃO GERAL}

Quando aplicada a duas amostras de crianças de 6 a 11 anos de idade, sendo a primeira amostra de 60 crianças normais (i.e., sem queixa de distúrbio de processamento auditivo central) e a segunda amostra de 11 crianças com diagnósti- co de distúrbio de processamento auditivo central, a bateria de ZAIDAN (2001) para triagem de distúrbio de processamento auditivo central mostrou-se válida e eficaz em discriminar entre os dois grupos de crianças, tanto em termos do escore total na bateria, quanto em termos dos escores individuais de cada um de seus três testes: $\mathrm{O}$ teste de fala filtrada, o teste de fala no ruído, e o teste de palavras competitivas.

Este artigo reanalisou os resultados do mesmo estudo a partir de estatísticas inferenciais paramétricas, mais precisamente, Ancovas seguidas de análises de comparação de pares. Controlando, assim, o efeito da idade das crianças como covariante, foi possível demonstrar claramente a eficácia da bateria toda em discriminar entre crianças com e sem queixa de distúrbio de processamento auditivo central. Contudo, é bom lembrar que isto só pôde ser feito devido ao meritório cuidado de ZAIDAN (2001) em fornecer os dados brutos em que se basearam asuas análises (ver Tabela 16). Sem tais dados, as presentes reanálises teriam sido impossíveis.

Este artigo demonstra que todos os três testes da bateria de triagem de Zaidan conseguiram, de fato, discriminar entre os grupos de maneira significativa e clara e que o teor inconclusivo dos achados de sua dissertação não se deveu a qualquer inadequação da bateria mas, sim, à inadequação da escolha das estatísticas empregadas para a análise de dados. Como a amostra do grupo experimental era bastante pequena $(\mathrm{N}=1 \mathrm{l}$ ) e apresentava uma grande variação etária (de 6 a 11 anos), não haveria praticamente nenhuma chance de poder demonstrar a sensibilidade do teste em identificar diferença significativa entre os grupos, a menos que a variabilidade dos desempenhos dentro desses grupos fosse controlada pelo emprego de análise de covarância tendo a idade como covariante, como foi feito neste artigo.

Assim, o presente artigo demonstrou não apenas a validade da bateria de Zaidan para triagem de processamento auditivo central como, também, a necessidade de usar estatísticas mais apropriadas às questões experimentais levantadas, de modo a obter respostas mais adequadas quanto à sensibilidade e validade de instrumentos tão importantes quanto a bateria de Zaidan para triagem de processamento auditivo central. Ressaltou, também a importância de que as dissertações e teses sempre forneçam os dados brutos em que se baseiam suas análises para permitir o constante avanço da ciência e a valorização plena de cada estudo experimental conduzido. 


\begin{abstract}
The paper defines central auditory processing and explains its importance to literacy acquisition. It explains the nature and etiology of central auditory processing disorders in a history of repeated episodes of otitis, as well as its involvement in phonological processing disorders that underlie problems in the development of oral and written language, as in some cases of developmental dyslexia. The paper highlights the need for instruments capable of screening children with central auditory processing disorders in order to assess the effectiveness of intervention procedures aimed at preventing and treating oral and written language disorders. The paper examines ZA I DAN’^S (2001 ) battery for screening central auditory processing disorders $\wedge 7$ which consists of three tests for assessing the capability of repeating words presented under difficult audibility conditions: speech distorted by the omission of high frequency sounds, speech presented against background noise, and competitive speech presented in dichotic listening. In the present study, data re-analyses were conducted, this time with appropriate inferential statistics: ANCOVAs in order to control for wide variation among age levels. Results showed that ZAIDAN's battery is, in fact, highly valid for 6-11- year-old children and sensitive enough to discriminate between children with and without clinical diagnosis of central audito $\sim \mathrm{y}$ processing disorder. Results showed that the validity and sensitivity were not res^b-icted to the battery as a whole but, rather, characterized each one of the three tests that compose it, since each test was, per se, sensitive enough to discriminate between children with and without central auditory processing disorders in the 6-1 1 age levels. Thus, Brazilian researchers, educators and practitioners have a powerful instrument for identifying children at risk of failing literacy acquisition, and for providing early intervention.
\end{abstract}

Key words: central auditory processing; learning disorder; dyslexia.

\section{REFERÊNCIAS BIBLIOGRÁFICAS}

ALVAREZ, A. M. M. A.; CAETANO, A. L.; NASTAS, S. S. Processamento auditivo central. O que e isto? Fono Atual, 1(1): 17-18, 1997.

AMERICAN SPEECH-LANGUAGE-HEARING ASSOCIATION. Central auditory processing current status of research and implications for clinical practice. American Journal of Audiology, 5(2): 41 -54, 1996.

AMOS, N. E.; HUMES, L. E. SCAN test-retest reliability for first and third-grade children. Journal of Speech-Language and Hearing Research, 41: 834-845, 1998.

BALEN, S. A. Processamento auditivo central: Aspectos temporais de audição e percepção acústica da fala. Sao Paulo, 1997. [Dissertaçção de Mestrado - Pontifícia Universidade Católica de São Paulo].

BARAN,.J. A.; MUSIEK, F. E. Behavioral assessment of the central auditory nervous system. In: F. E. MlJSIEK \& W. F. RINTELMANN (Eds.), Contempory perspectives in hearing assessment (pp. 375-413). Boston, MA: Allyn \& Bacon, 1999.
BELLIS, T. J. Assessment and management of central auditory processing disorder in the educational setting. San Diego, CA: Singular, 1996.

BERLIN, C. l.; LOWE-BELL, S. S.; JANNETTA, P. J.; KLINE, D. G. Central auditory deficits after temporal lobectomy. Archives of Otolaryngology, 96: 4-10, 1972.

BESS, F. H.; HULMES. L. E. Fundamentos de audiologia. $2^{\mathrm{a}}$ ed. Porto Alegre, RS: Artes Médicas, 1998. p. 196-229.

BORGES, A. C. L. C. Percepção auditiva para deficientes auditivos com palavras - MTS. In: L.D. PEREIRA \& E. SCHOCHAT (Eds.), Processamento auditivo central. Manuul de avaliação. São Pau1o, SP: Lovise, 1997a. p. 197-208.

BORGES, A. C. L. C. Dissílabos alternados SSW. In L. D. PEREIRA \& E. SCHOCHAT (Eds.), Processamento auditivo central. Manual de avaliação. São Paulo, SP: Lovise, 1997b. p.169-178.

BRANDLS, P. J.; EHRINGER, D. M. The effects of early middle ear pathology on auditory perception and academic achievement. Journal of Speech and Hearing Disorders, 46(3): 301-306, 1981. 
CAI'OVILLA, F. C.; CAPOVILLA, A. G. S. Compreendendo a natureza dos problemas de aquisição de leitura e escrita: Mapeando o envolvimento de distúrbios cognitivos de discriminação fonológica, velocidade de processamento e memória fonológica. Cadernos de Psicopedagogia, I(1): 14-37, 2001.

CARVALHO, R. M. M. Processamento auditivo: Avaliação audiologica básica. In: L. D. PEREIRA \& E. SCHOCHAT (Eds.), Processamento auditivo central: Manual de avaliação. Sao Paulo, SP: Lovise, 1997. p. 27-35.

CARVALHO, I. A. M.; ALVAREZ, A. M. M. A.; CAETANO, A. L. Perfil de habilidades fonológicas. Sao Paulo, SP: Via Lettera, 1998.

CHERMAK, G. D.; MUSIEK, F. E. Central auditory processing disorders. New perspectives. San Diego, CA: Singular Publishing, 1997.

CHERMAK, G. D.; MUSIEK, F. E. Managing central auditory processing disorders in children and youth. American Journal of Audiology, 1(3): 61-65, 1992.

CHERMAK, G. D.; TRAYNHAM, W. A.; SEIKEI.., J. A.; MUSIEK, F. E. Professional education and assessment practices in central auditory processing. Journal of the American Academy of Audiology, 9: 452-465, 1998.

CHERRY, R. Screening and evaluation of central auditory processing disorders in young children. In: J. Katz, N.A. Stecker \& D. Henderson (Eds.), Central auditory processing. A trunsdisciplinary view. Chicago, IL: Mosby Year Book, 1992. p. 129-140.

COSTA, M. J.; IORIO, M. C. M.; MANGABEIRA ALBERNAZ, P. L. M. Reconhecimento de fala: Desenvolvimento de uma lista de sentenças em português. Acta Who, 16(4): 164-173, 1997a.

COSTA, M. J.; IORIO, M. C. M.; MANGABEIRAALBERNAZ, P. L. M. Sentencas para avaliar reconhecimento de fala. In: L.D. Pereira \& E. Schochat (Eds.), Processamento auditivo centrul. Manual de avaliação. São Paulo, SP: Lovise, 1997b. p. 181-185.

CRUZ, P. C.; PEREIRA, L. D. Comparação do desempenho das habilidades auditivas e de linguagem em crianças com queixa de dificuldade de aprendizagem. Acta W/IO, 15(1): $21-26,1996$.

DAVIS, S. M.; MCCROSKEY, R. L. Auditory fusion in children. Child Development, 51: 75-80,1980.

DOWNS, M. P. Effects of mild hearing loss on auditory processing. Otolaryngologic Clinics of North America, 18: 337-344, 1985.
FERRE, J. M. Processing power. A guide to central auditory processing disorder assessment and management. San Antonio, TX: Communication Skill Builders, 1997.

GORDON, N.; WARD, S. Abnorrnal response to sound and central auditory processing disorder. Developmental Medicine and Child Neurology, 37: 645-652, 1995.

GRAVEL, J. S.; WALLACE, J. F. Listening and language at four years of age: Effects of early otitis media. Journal of Speech-Language and Hearing Research, 35: 588-595, 1992.

HOOD, L. J.; BERLIN, C. I. Central auditory function disorders. In: J. L. NORTHERS (Ed.), Hearing disorders. Boston, MA: Allyn Y Bacon, 1996. p. 227-243.

KALIL, D. M.; ZILIOTTO, K. N.; ALMEIDA, C. I. R. SSI em portugues. In L. D. PEREIRA \& E. SCHOCHAT (Eds.), Processamento auditivo central. Manual de avaliação. São Paulo, SP: Lovise, 1997. p. 129-136.

KATZ, J. Classification of auditory processing disorders. In: J. KATZ, N. A. STECKER \& D. HENDERSON (Eds.), Central auditory processing. A transdisciplinary view. St. Louis, MO: Mosby Yearbook, 1992.

KATZ, I.; WILDE, L. Distúrbios da percepção auditiva em crianças. In: :1. KATZ (Ed.), Tratado de audiologia clínica (3 ${ }^{\mathrm{a}}$ ed., pp. 674-694). São Paulo, SP: Manole, 1989.

KEITH, R.W. SCAN: A screening test for central auditory processing clisorders. San Antonio, TX: The Psychological Corporation, 1986.

KEITH, R.W. Tests of central auditory function. In: R. ROESER \& M.P. DOWNS (Eds)., Auditory cli.sorders in school children. Identification and remediation. New York, NY: Thieme Medical, 1988. p. 83-97.

KEITH, R. W.; PENSAK, M. L. Central auditory function. Otolaringologic Clinics of North America, 24: 371-379, 1991.

KRAUS, N.; MCGEE, T.; CARREL, T.; SHARMA, A. Neurophysiologic bases of speech discrimination. Earl and Hearing, 16(1): 19-37, 1995.

KRAUS, N.; MCGEE, T.; CARREL, T.; ZECKER, N.; NICOL, T.; KOCH, D. Auditory neurophysiologic responses and discrimination deficits in children with learning problems. Science, 273: 971-973, 1996.

LEWIS, M. Learning disabilities and prenatal risks. Urbana, IL: University oflllinois Press, 1996.

MAC FARLAND, D. J.; CACACE, A. T. Central auditory processing disorder in school-aged children: A critical review. Journal of SpeechLanguage and Hearing Research, 41: 355-373, 1998. 
MAC FARLAND, D. J; CACACE, A.T. Modality specificity as a criterion for diagnosing central audotory processing disorders. American Journal of Audiology, 26(3): 36-48, 1995.

MACHADO, S. F. Percepção da fala. Fundamentos para terapia e avaliação. São Paulo SP: Plexus, 1996.

MUSIEK, F. E.; BARAN, J. Central auditory assessment: 30 years of challenge and change. Ear and Hearing, 8: 22S-35S, 1987.

MUSIEK, F. E.; LAMB, L. Avaliação auditiva central: Uma visão geral. In: J. KATZ (Ed.), Tratado de audiologia clínica. $4^{\mathrm{a}}$ ed. São Paulo, SP: Manole, 1999. p. 195-209.

MUSIEK, F. E.; KURDZIEL-SCHWAN, S.; KIBBE, K. S.; GOLLEGLY, K. M.; BARAN, J. A.; RINTEI.MANN, W. F. The dichotic rhyme task: Results in splitbrain patients. Ear and Hearing, 10: 33-39, 1989.

ORTIZ, K. Z.; PEREIRA, L. D. Não-verbal de escuta direcionada. In: L. D. PEREI RA \& E. SCHOCHAT (Eds.), Processamento auditivo central. Manual de avaliação. São Paulo, SP: Lovise, 1997. p. 151-157.

PEREI RA, L. D. Audiometria verbal: Teste de discrimina,cao vocal com ruído. São Paulo, 1993. [Tese de Doutorado - Universidade Federal de Sao Paulo].

PEREIRA, L. D.; SCHOCHAT, E. Baixa redundância: Fala filtrada e fusão biaural. In: L. D. PERF.IRA \& E. SCHOCHAT (Eds.), Processamento auditivo central. Manual de avaliação. São Paulo, SP: I.ovise, 1997a. p. 103-109.

PEREIRA, L. D.; SCHOCHAT, E. (Eds.). Processamento auditivo central. Manuul de avaliação. Sao Paulo, SP: Lovise, 1997b.

PHILLIPS, D. P. Central auditory processing: A view from auditory neuroscience. The American Journal of Otology, 16(3): 338-352, 1995.
PILLSBURY, H. C.; GROSE, J.H.; HALL, J. W. Otitis media with effusion in children. Archives of Otolaringology, Heacl und Neck Surgery, 117: 717-783, 1991.

SANTOS, M. F. C. S.; PEREIRA, L. D. Escuta com dígitos. In: L. D. PEREIRA \& E. SCHOCHAT (Eds.), Processamento auditivo central. Manual de avaliação. São Paulo, SP: Lovise, 1997. p. 147-15().

SANTOS, M. T. M. S.; PEREIRA, L. D. Consciência fonológica. In: L.D. PEREIRA \& E. SCHOCHAT (Eds.), Processamento auditivo central. Manual de avaliação. São Paulo, SP: Lovise. 1997. p. 187-195.

SCHOCHAT, E. Sons ambientais competitivos CES. In: L. D. PEREIRA \& E. SCHOCHAT (Eds.), Processamento auditivo central. Manual de avaliação. São Paulo, SP: Lovise, 1997. p. 159-167.

SCHOCHAT, E.; PEREIRA, L. D. Fala com ruído. In: L. D. PEREIRA \& E. SCHOCHAT (Eds.), Processamento auditivo central. Manual de avaliação. São Paulo, SP: Lovise, 1997. p.99-102.

TEDESCO, M. L. F. Consoante-vogal de escuta direcionada. In: L.D. PEREIRA \& E. SCHOCHAT (Eds.), Processamento auditivo central: Manual de avaliação. São Paulo, SP: Lovise, 1997. p.139-145.

ZAIDAN, E. Desenvolvimento de uma bateria de testes de triagem da funcão auditiva central em preescolares e escolares na faixa de 6 a 11 anos. São Paulo, 2001. [Disserta,cao de mestrado - Universidade de São Paulo].

ZILIOTTO, K. N.; KALIL, D. M.; ALMEIDA, C. I. R. PSI em português. In: L. D. PEREIRA \& E. SCHOCHAT (Eds.), Processamento auditivo central. Manual de avaliação. São Paulo, SP: Lovise, 1997. p. 113-128.

Recebido em 30/07/2002 Aprovado em 12/08/2002 